\title{
Behavioral and Physiological Effects of a Novel Kappa-Opioid Receptor-Based DREADD in Rats
}

\author{
Nathan J Marchant ${ }^{* 1,2,7}$, Leslie R Whitaker',7, Jennifer M Bossert ${ }^{1,7}$, Brandon K Harvey ${ }^{3}$, Bruce T Hope', \\ Konstantin Kaganovsky', Sweta Adhikary', Thomas E Prisinzano ${ }^{4}$, Eyal Vardy, ${ }^{5,6,7}$, Bryan L Roth ${ }^{6,7}$ and \\ Yavin Shaham ${ }^{*, 1,7}$ \\ 'Behavioral Neuroscience Research Branch, IRP-NIDA, NIH, Bethesda, MD, USA; ${ }^{2}$ Florey Institute of Neuroscience and Mental Health, University \\ of Melbourne, Parkville, VIC, Australia; ${ }^{3}$ Optogenetics and Transgenic Technology Core, IRP-NIDA, NIH, Bethesda, MD, USA; ${ }^{4}$ Department of \\ Medicinal Chemistry, School of Pharmacy, University of Kansas, Lawrence, KS, USA; ${ }^{5}$ Merck Research Laboratories, Kenilworth, NJ, USA; \\ ${ }^{6}$ Department of Pharmacology, School of Medicine, University of North Carolina Chapel Hill, Chapel Hill, NC, USA
}

In the past decade, novel methods using engineered receptors have enabled researchers to manipulate neuronal activity with increased spatial and temporal specificity. One widely used chemogenetic method in mice and rats is the DREADD (designer receptors exclusively activated by designer drugs) system in which a mutated muscarinic $\mathrm{G}$ protein-coupled receptor is activated by an otherwise inert synthetic ligand, clozapine-N-oxide (CNO). Recently, the Roth laboratory developed a novel inhibitory DREADD in which a mutated kappa-opioid receptor (KORD) is activated by the pharmacologically inert drug salvinorin B (SalB; Vardy et al, 20I 5). They demonstrated the feasibility of using KORD to study brain circuits involved in motivated behavior in mice. Here, we used behavioral, electrophysiological, and neuroanatomical methods to demonstrate the feasibility of using the novel KORD to study brain circuits involved in motivated behavior in rats. In Exp. I, we show that SalB dose-dependently decreased spontaneous and cocaine-induced locomotor activity in rats expressing KORD to midbrain (ventral tegmental area/substantia nigra). In Exp. 2, we show that SalB completely inhibited tonic firing in KORDexpressing putative dopamine neurons in midbrain. In Exp. 3, we used a 'retro-DREADD' dual-virus approach to restrict expression of KORD in ventral subiculum neurons that project to nucleus accumbens shell. We show that KORD activation selectively decreased novel context-induced Fos expression in this projection. Our results indicate that the novel KORD is a promising tool to selectively inactivate brain areas and neural circuits in rat studies of motivated behavior.

Neuropsychopharmacology (2016) 4I, 402-409; doi:I0.1038/npp.20I5.I49; published online 24 June 2015

\section{INTRODUCTION}

In the past decade, several novel optogenetic and chemogenetic techniques using engineered receptors have enabled researchers to manipulate neuronal activity with increased spatial and temporal specificity (Boyden et al, 2005; Armbruster et al, 2007). Chemogenetics refers to the approach of using engineered receptors that can inhibit (Armbruster et al, 2007) or activate (Alexander et al, 2009) neurons when an otherwise inert ligand binds to them (Urban and Roth, 2015). DREADDs (designer receptors exclusively activated by designer drugs) are chemogenetic tools with therapeutic utility (Urban and Roth, 2015) and have been used to manipulate cell signaling within diverse cell types (Rogan and Roth, 2011). However, the dependency

* Correspondence: Dr N Marchant or Dr Y Shaham, Behavioral Neuroscience Research Branch, IRP-NIDA, NIH, Bethesda, MD, USA, Tel: + I 443740 2704, Fax: + I 4437402827 ,

E-mail: nathan.marchant@nih.gov or Yshaham@intra.nida.nih.gov

${ }^{7}$ These authors contributed equally to this work.

Received 23 March 20I5; revised I May 20I5; accepted 20 May 20I5; accepted article preview online 28 May 2015 on the same chemical actuator clozapine- $N$-oxide $(\mathrm{CNO})$ has restricted the ability to use DREADDs to selectively either inhibit or activate neuronal activity in the same animal.

Recently, the Roth laboratory developed a novel DREADD that is based on the Gi-coupled kappa-opioid receptor (KORD) and the pharmacologically inert ligand is salvinorin B (SalB) (Vardy et al, 2015). SalB is a metabolite of the KOR selective agonist salvinorin A (SalA) that has minimal or no affinity to KOR and numerous other receptors, as assessed in receptor binding assays (Roth et al, 2002; Ansonoff et al, 2006). SalB also has minimal or no observed behavioral effects in mice under conditions in which SalA is highly effective (eg, increases in brain stimulation threshold) (Vardy et al, 2015). In the paper describing the development and validation of KORD, Vardy et al used mice to show that KORD-mediated inhibition of ventral tegmental area (VTA) GABAergic neurons dose-dependently increased locomotion, and that KORD-mediated inhibition of hypothalamic arcuate nucleus Agouti-Related Peptide (AgRP) decreased food intake, whereas paraventricular SIM1 neuron inhibition increased food intake. These studies show that KORD is effective at changing certain behaviors in mice. Here, we 
validate the use of KORD in rats because our goal is to eventually use KORDs in circuitry-related studies that control appetitive and aversive motivated behaviors, studies that cannot be performed or are very difficult to perform in mice (see Discussion).

Here, we demonstrate the feasibility of KORD as a tool to study brain circuits of motivated behaviors in rats using behavioral, electrophysiological, and neuroanatomical approaches. We show that SalB dose-dependently decreased spontaneous and cocaine-induced locomotor activity in rats expressing KORD in midbrain. We also show that SalB inhibited tonic firing in KORD-expressing putative dopamine neurons in midbrain. These effects were selective for the specific combination of DREADD and ligand, because SalB had no apparent effect on locomotor activity or neuronal firing in rats bilaterally injected with the Gi/oDREADD hM4Di. In addition, we describe a dual-virus method to restrict DREADD expression to projection neurons in long-range circuits (Boender et al, 2014; Nair et al, 2013; Rothermel et al, 2013; Friedman et al, 2014) that we term herein 'retro-DREADD.' Using novel context exposure to increase neural activity, indexed by Fos expression (Morgan and Curran, 1991), we show that SalB decreased Fos expression in ventral subiculum neurons that project to nucleus accumbens shell (Groenewegen et al, 1987; Pennartz et al, 1994). These data demonstrate the ability of KORD to inhibit neural activity in vivo, in a projectionspecific manner, in rats. This method may be especially useful for investigators interested in studying the causal role of defined neural projections in complicated behaviors where the experimental subjects are rats.

\section{MATERIALS AND METHODS}

\section{Subjects}

We used male Sprague-Dawley rats (Charles River or NIDA breeding core, $n=49$ ) weighing $250-450 \mathrm{~g}$ before surgery. The rats in the electrophysiology experiment were previously tested in the locomotor activity experiments. We maintained the rats under a reverse $12: 12 \mathrm{~h}$ light/dark cycle with food and water freely available. We housed the rats two per cage before and after surgery. We performed the experiments in accordance with the National Institutes of Health Guide for the Care and Use of Laboratory Animals (8th edition) under protocols approved by the Animal Care and Use Committee. We excluded seven rats due to unilateral, low, or no virus expression.

\section{Viral Injection Surgery}

We anesthetized the rats with ketamine and xylazine (80 and $10 \mathrm{mg} / \mathrm{kg}$, i.p., respectively) and used $10 \mu \mathrm{l}$ microinjection syringes (Hamilton syringe or Nanofil from World Precision Instruments) with 33 gauge needles secured to stereotaxic pumps (UMP4 injector; World Precision Instruments). We set the nose bar at $-3.3 \mathrm{~mm}$. In Exp. 1 and 2, we bilaterally injected AAV9.CaMKIIa.HA-KORD.IRES.mCitrine ( $A A V$ $K O R D$; titer $3.0 \times 10^{12} \mathrm{gc} / \mathrm{ml}$; synthesized by $\mathrm{BR}$ and $\mathrm{EV}$, UNC vector core) or AAV1.CamKIIa.HA-hM4D(Gi).IRES. mCitrine $\left(A A V-h M 4 D i\right.$; titer $1.39 \times 10^{12} \mathrm{gc} / \mathrm{ml}$; synthesized by BH, OTTC, IRP-NIDA) into VTA/substantia nigra (midbrain) using the following coordinates from Bregma
(Paxinos and Watson, 2008): AP: $-5.2 \mathrm{~mm}$; ML: $\pm 3.0 \mathrm{~mm}$ $\left(10^{\circ}\right.$ angle); and DV: $-8.5 \mathrm{~mm}$.

In Exp. 3, we unilaterally injected AAV5.CMV.HI.eGFPCre.WPRE.SV40 (AAV-Cre; titer $8.56 \times 10^{12} \mathrm{gc} / \mathrm{ml}$; UPenn vector core) into nucleus accumbens shell (AP: $+1.6 \mathrm{~mm}$, ML: $\pm 2.3 \mathrm{~mm}\left(10^{\circ}\right.$ angle), DV: $\left.-7.5 \mathrm{~mm}\right)$ and unilaterally injected AAV9.SYN1.dF.HA.KORD.IRES.mCitrine ( $A A V$ DIO-KORD; titer $1.2 \times 10^{13} \mathrm{gc} / \mathrm{ml}$; synthesized by $\mathrm{BR}$ and EV, UNC vector core) into ipsilateral ventral subiculum (AP: $-6.0 \mathrm{~mm}, \mathrm{ML}: \pm 5.3 \mathrm{~mm}$ ( $4^{\circ}$ angle), DV: $-8.5 \mathrm{~mm}$ ). These coordinates are based on our previous studies (Bossert et al, 2004, 2012; Bossert and Stern, 2014).

For all surgeries, we injected $0.75 \mu \mathrm{l}$ of the virus solution per side at a rate of $0.25 \mu \mathrm{l} / \mathrm{min}$ over $3 \mathrm{~min}$ and we left the microinjector needles in place for $1 \mathrm{~min}$ before removing them. We then placed bonewax (Butler Schein) over the holes, sutured the wounds, and removed the rats from the stereotaxic frame. We injected rats with $3 \mathrm{ml}$ sterile $0.9 \%$ saline (s.c.) to prevent dehydration and injected rats with the NSAID ketoprofen $(2.5 \mathrm{mg} / \mathrm{kg}$, s.c.) immediately after surgery and the day after surgery to reduce inflammation. We handled the rats three times per week before the start of the locomotor tests (began $2 \frac{1}{2}-3$ weeks after surgery) or before the homecage vs novel context exposure (6 weeks after surgery).

\section{Drugs}

We obtained (- )-cocaine hydrochloride (dissolved in sterile saline) from NIDA and injected it at a dose of $10 \mathrm{mg} / \mathrm{kg}$ (i.p.). Salvinorin B (SalB) was synthesized in the laboratory of Thomas E. Prisinzano at the University of Kansas, as described previously (Tidgewell et al, 2004) and dissolved in $100 \%$ DMSO at doses of $0,7.5,15$, and $30 \mathrm{mg} / \mathrm{kg}$ (s.c.). We chose these doses based on the recent mouse study (Vardy et al, 2015) and on a pilot experiment in which we determined that these doses of SalB had no effect on spontaneous locomotion and cocaine-induced locomotion in rats not injected with virus (data not shown). We obtained $\mathrm{CNO}$ from the NIH Investigative Drug Program and dissolved it in $5 \%$ DMSO in sterile water. We injected it at a dose of $3 \mathrm{mg} / \mathrm{kg}$ (i.p.). This dose is based on the previous reports (Ferguson et al, 2011, 2013; Nair et al, 2013).

\section{Specific Experiments}

Exp. 1: Behavioral Validation of KORD. We tested whether SalB would decrease spontaneous and cocaineinduced locomotor activity in rats $(n=8)$ injected bilaterally with AAV-KORD into midbrain (Figure 1a). For this purpose, we used an experimental design that included the within-subject factors of SalB Dose (0 (DMSO), 7.5, 15, and $30 \mathrm{mg} / \mathrm{kg}$ ) and Cocaine Dose (0 (saline), $10 \mathrm{mg} / \mathrm{kg}$ ). We used locomotor chambers (Accuscan Instruments; $42 \times 42 \times$ $30 \mathrm{~cm}^{3}$ ) and collected data in 5-min intervals using the VersaMax version 3.0 data analysis system (Accuscan Instruments). We used total distance traveled to evaluate the effect of SalB on spontaneous and cocaine-induced locomotor activity. We first placed the rats in the locomotor boxes for $30 \mathrm{~min}$. We then injected them with the SalB dose and $10 \mathrm{~min}$ later we injected them with either saline or 
a

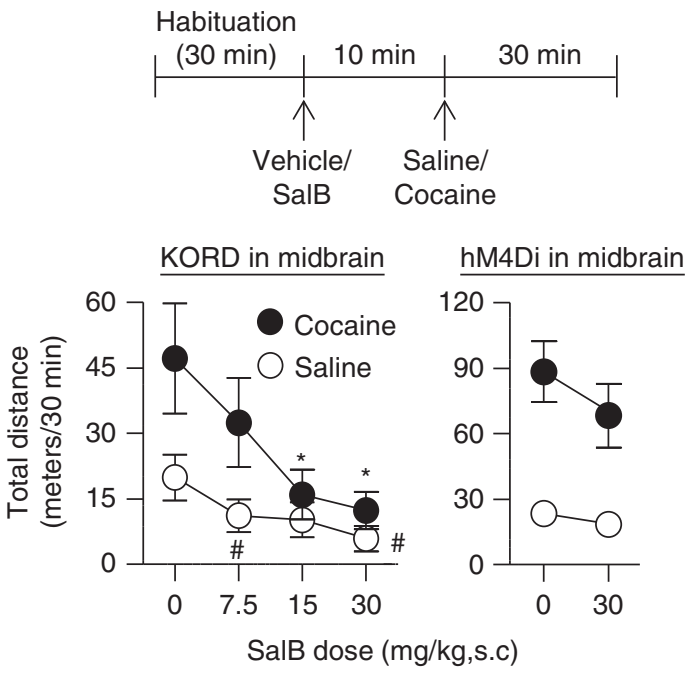

b
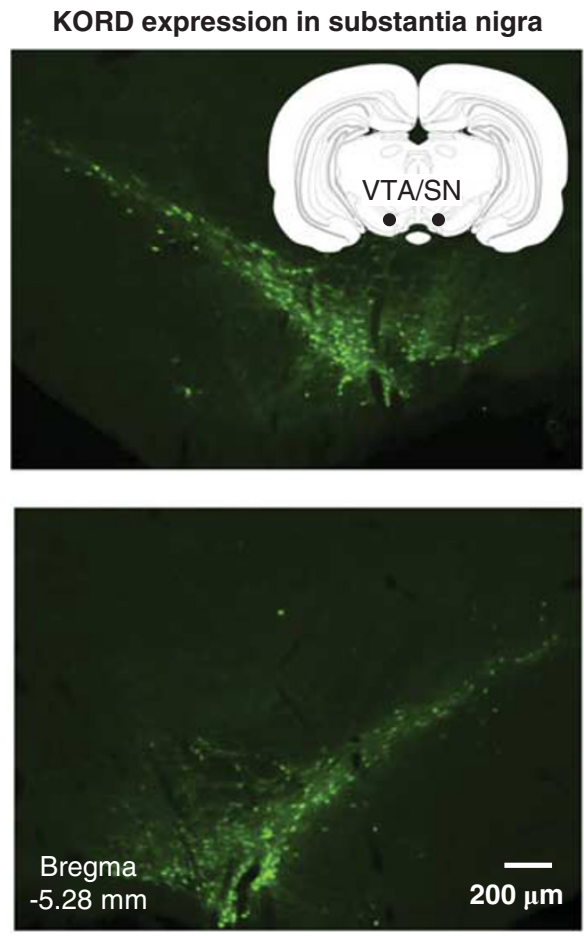

\section{Effect of SalBon firing rate}

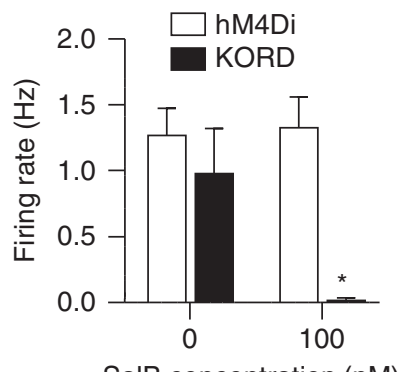

SalB concentration $(\mathrm{nM})$

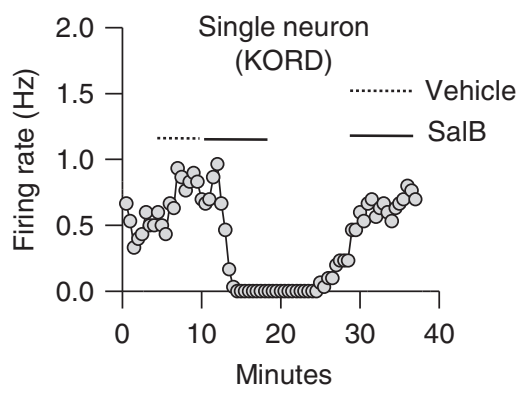

d
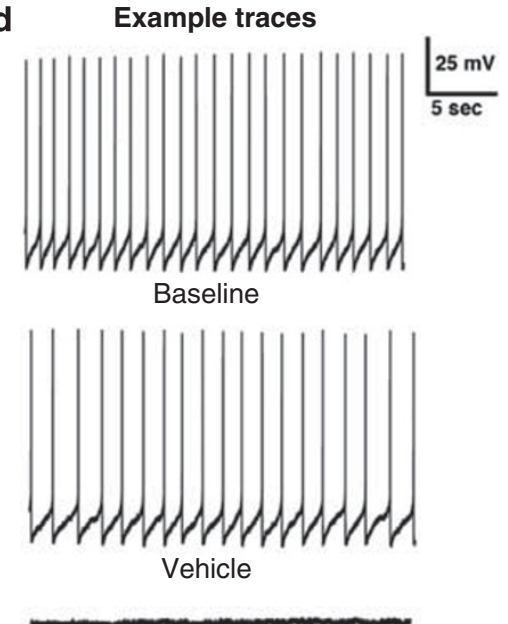

SalB

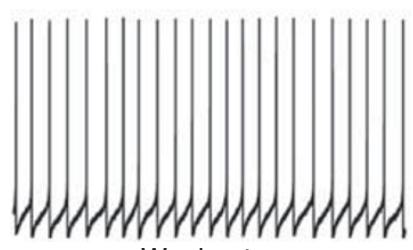

Washout

Figure I Effect of Salvinorin B on locomotor activity and putative dopamine cell firing in rats expressing KORD in midbrain. (a) Top: Timeline of the locomotor experiment. Bottom: Mean \pm SEM total distance in meters in rats expressing either KORD (left; $n=8)$ or $h M 4 D($ right; $n=1 \mathrm{l}$ ) in midbrain. * Different from vehicle in the Cocaine condition, $P<0.05$; ${ }^{*}$ Different from vehicle in the Saline condition, $P<0.05$. (b) Representative images of bilateral KORD (native mCitrine) expression in midbrain. Scale $=200 \mu \mathrm{m}$. VTA, ventral tegmental area; SN, substantia nigra. (c) Top: Firing rate (Hz) of putative midbrain dopamine neurons expressing either KORD (2 rats, 5 neurons) or hM4i-DREADD ( 3 rats, 10 neurons) after administration of vehicle or SalB (I $00 \mathrm{~nm})$. *Different from the other experimental conditions, $P<0.05$. Bottom: Time course from an example experiment in which SalB inhibited tonic firing in a single putative dopamine neuron. Firing returned to baseline rate after drug washout. (d) Example traces from KORD-expressing neurons after vehicle and SalB bath application.

cocaine and measured locomotor activity for $30 \mathrm{~min}$; we counterbalanced the order of the injections of the different doses of SalB and saline/cocaine during the 4-test daily test sessions.
To determine selectivity of SalB to KORD, we also measured spontaneous and cocaine-induced locomotor activity in rats $(n=11)$ injected with AAV-hM4Di into midbrain; we chose this virus as the control condition because its other 
components (CamKIIa, IRES, and mCitrine) are similar to those of AAV-KORD (albeit with different serotypes). We evaluated the effect of SalB on locomotor activity using an experimental design that included the within-subject factors of SalB Dose $(0,30 \mathrm{mg} / \mathrm{kg})$ and Cocaine Dose $(0,10 \mathrm{mg} / \mathrm{kg})$. Finally, we repeated the experiment in most of the rats $(n=9)$ where we injected vehicle or $\mathrm{CNO}(3 \mathrm{mg} / \mathrm{kg}$, i.p.) $30 \mathrm{~min}$ before saline or cocaine injections during four daily locomotor activity sessions.

To verify expression of KORD in midbrain, we deeply anesthetized three AAV-KORD-injected rats (the other rats were used in Exp. 2) with isoflurane (approximately $90 \mathrm{~s}$ ) and perfused them transcardially with $100 \mathrm{ml}$ of $0.1 \mathrm{M}$ phosphate-buffered saline (PBS) followed by $400 \mathrm{ml}$ of $4 \%$ paraformaldehyde (PFA) in $0.1 \mathrm{M}$ sodium phosphate $(\mathrm{pH}$ 7.4). We removed the brains and post-fixed them in 4\% PFA for $2 \mathrm{~h}$ before we transferred them to $30 \%$ sucrose in $0.1 \mathrm{M}$ sodium phosphate ( $\mathrm{pH} 7.4$ ) for $48 \mathrm{~h}$ at $4{ }^{\circ} \mathrm{C}$. We subsequently froze the brains in powdered dry ice and used a cryostat to collect coronal sections $(40 \mu \mathrm{m})$ containing midbrain (approximately -4.8 to $-5.6 \mathrm{~mm}$ from Bregma) in $0.1 \%$ sodium azide. We digitally captured dark-field images of native mCitrine fluorescence in the midbrain using an EXi Aqua camera (QImaging) attached to a Zeiss Axio Scope 2, Axio Imager M2. We captured and analyzed the images using iVision (Biovision).

Exp. 2: Electrophysiological Validation of KORD. The electrophysiology of this procedure is based on a previous study (Whitaker et al, 2013). At least 3 days after the rats completed the locomotor activity experiments, we deeply anesthetized them with isoflurane (60-90 s) and then rapidly decaptitated them. We cut horizontal midbrain slices in icecold solution containing (in mM) 92 NMDG, 20 HEPES, 25 Glucose, $30 \mathrm{NaHCO}_{3}, 1.2 \mathrm{NaH}_{2} \mathrm{PO}_{4}, 2.5 \mathrm{KCl}, 5 \mathrm{Na}$-ascorbate, 3 Na-pyruvate, 2 Thiourea, $10 \mathrm{MgSO}_{4}, 0.5 \mathrm{CaCl}_{2}$, saturated with $95 \% \mathrm{O}_{2} 5 \% \mathrm{CO}_{2}(\mathrm{pH} \mathrm{7.3-7.4,} 305 \mathrm{mOsm} / \mathrm{kg})$. We then incubated the slices for $5 \mathrm{~min}$ at $35^{\circ} \mathrm{C}$ in the same solution. We allowed the slices to recover for a minimum of $30 \mathrm{~min}$ at room temperature in artificial cerebrospinal fluid (ACSF) containing (in $\mathrm{mM}$ ) $126 \mathrm{NaCl}, 2.5 \mathrm{KCl}, 1.2 \mathrm{MgCl}_{2}$, $2.4 \mathrm{CaCl}_{2}, 1.2 \mathrm{NaH}_{2} \mathrm{PO}_{4}, 21.4 \mathrm{NaHCO}_{3}, 11.1$ Glucose, $3 \mathrm{Na}$ pyruvate, and $1 \mathrm{Na}$-ascorbate. We performed the recordings at $32-35^{\circ} \mathrm{C}$ in the same solution after adding $50 \mu \mathrm{M}$ picrotoxin (to block $\mathrm{GABA}_{\mathrm{A}}$ receptors), which was bathperfused at $2 \mathrm{ml} / \mathrm{min}$. Intracellular solution contained (in $\mathrm{mM}$ ) $115 \mathrm{~K}$-gluconate, $20 \mathrm{KCl}, 1.5 \mathrm{MgCl}_{2}, 10 \mathrm{HEPES}$, 0.025 EGTA, 2 Mg-ATP, $0.2 \mathrm{Na}_{2}$-GTP, $10 \mathrm{Na}_{2}$-phosphocreatine $(\mathrm{pH} \mathrm{7.2-7.3,} 285 \mathrm{mOsm} / \mathrm{kg})$.

We identified DREADD-expressing (mCitrine-positive) neurons using a scanning disk confocal microscopy (Olympus FV1000) and used differential interference contrast optics to patch neurons. We performed whole-cell recordings in electrophysiologically identified putative dopamine neurons of midbrain pars compacta in zero current mode (Grace, 1988; Morikawa et al, 2003; Ungless and Grace, 2012). We identified putative dopamine neurons by their spontaneous firing $(0.5-5 \mathrm{~Hz})$ with broad APs (>1.2 ms) and large $I_{h}$ currents ( $>200$ pA elicited by 1.5 s hyperpolarizing step from $-60 \mathrm{mV}$ to $-110 \mathrm{mV}$ ) recorded in whole-cell configuration (Whitaker et al, 2013). We discarded recording if the series resistance change was $>10 \%$ during the course of the recording, or if input resistance fell below $180 \mathrm{M} \Omega$. We used an Axopatch 200B amplifier (Molecular Devices) and Axograph X software (Axograph Scientific) to record and collect the data, which we filtered at $2-10 \mathrm{kHz}$ and digitized at $4-20 \mathrm{kHz}$.

We applied SalB $(100 \mathrm{nM})$ and CNO $(10 \mu \mathrm{M})$ by superfusion to the slice (Krashes et al, 2011; Vardy et al, 2015). For AAV-KORD-injected rats, each recording session started with 5-10 min of baseline recording, following by superfusion of vehicle (DMSO) for 5-10 min, and SalB for 5-10 min. For $2 \mathrm{hM} 4 \mathrm{Di}$-injected rats (6 neurons), we followed the same procedure, but after SalB perfusion, we also superfused CNO for 5-10 min, followed by a 10 - to 20-min washout period.

Exp. 3: Immunohistochemical Validation of KORD in Combination with 'retro-DREADD'. We tested whether SalB would inhibit novel context-induced Fos in ventral subiculum neurons that project to nucleus accumbens shell. For this purpose, we used four groups of rats injected with AAV-Cre into nucleus accumbens shell and AAV-DIOKORD in ventral subiculum. We used a $2 \times 2$ factorial design that includes the between-subject factors of Context (home, novel) and SalB Dose (0 (vehicle), $15 \mathrm{mg} / \mathrm{kg}$ ). Six weeks after surgery, we injected rats with vehicle or $15 \mathrm{mg} / \mathrm{kg} \mathrm{SalB}$ and placed them in their homecage or a novel context (large round plastic bowel with bedding and toys). We returned the novel context rats to their homecage after $30 \mathrm{~min}$ because the effect of SalB on locomotor activity in mice lasts for less than 60 min (Vardy et al, 2015).

Ninety minutes after vehicle or SalB injection, we deeply anesthetized the rats with isoflurane (approximately $90 \mathrm{~s}$ ) and perfused them transcardially with $100 \mathrm{ml}$ of $0.1 \mathrm{M}$ PBS followed by $400 \mathrm{ml}$ of $4 \%$ PFA in $0.1 \mathrm{M}$ sodium phosphate $(\mathrm{pH} 7.4)$. We removed the brains and post-fixed them in 4\% PFA for $2 \mathrm{~h}$ before we transferred them to $30 \%$ sucrose in $0.1 \mathrm{M}$ sodium phosphate ( $\mathrm{pH} 7.4$ ) for $48 \mathrm{~h}$ at $4{ }^{\circ} \mathrm{C}$. We subsequently froze the brains in powdered dry ice and used a cryostat to collect coronal sections $(40 \mu \mathrm{m})$ containing nucleus accumbens shell (approximately +2.0 to $+1.0 \mathrm{~mm}$ from Bregma) and ventral subiculum (approximately -5.4 to $-6.4 \mathrm{~mm}$ from Bregma) in $0.1 \%$ sodium azide.

We rinsed free-floating sections three times for $10 \mathrm{~min}$ each in PBS, incubated for $2 \mathrm{~h}$ in $4 \%$ bovine serum albumin (BSA) in PBS with $0.4 \%$ Triton X-100 (PBS-Tx), and incubated overnight at $4{ }^{\circ} \mathrm{C}$ with rabbit anti-Fos monoclonal primary antibody (Phospho-c-Fos, 5348S, Cell Signaling) diluted 1:2000 and mouse anti-HA monoclonal primary antibody (HA.11 clone 16B12, MMS-101R, Covance) diluted $1: 1000$ in $4 \%$ BSA in PBS-Tx. We then rinsed sections in PBS and incubated for $2 \mathrm{~h}$ with AF 594 anti-rabbit secondary antibody (711-585-152, Jackson Immunoresearch) and AF 488 anti-mouse secondary antibody (A-11001, Invitrogen) diluted $1: 500$ in $4 \%$ BSA in $0.4 \%$ PBS-Tx. We then rinsed the sections again, mounted the tissue onto gelatin-coated slides, and coverslipped with Vectashield Hardset Mounting Medium (H-1400, Vector Labs).

We digitally captured dark-field images of native Cre-GFP fluorescence in nucleus accumbens shell and double labeling of Fos and HA (a tag fused to the KORD, here referred to as 
KORD) in ventral subiculum using an EXi Aqua camera attached to a Zeiss Axio Scope 2, Axio Imager M2 and analyzed the images using iVision (Biovision) in a doubleblind manner. We counted the number of Fos-immunoreactivity (IR), KORD-IR, and double-labeled (Fos+KORD) cells from three injected hemispheres of ventral subiculum and used the mean of the counts for the statistical analysis.

\section{Statistical Analyses}

Locomotor activity. We analyzed the data with the SPSS statistical software (IBM) using repeated measures ANOVA: within-subject factors of SalB dose $(0,7.5,15$, and $30 \mathrm{mg} / \mathrm{kg}$ for KORD, or 0 and $30 \mathrm{mg} / \mathrm{kg}$ for hM4Di) or CNO dose $(0$ and $3 \mathrm{mg} / \mathrm{kg}$ for $\mathrm{hM} 4 \mathrm{Di}$ ) and cocaine dose $(0$ and $10 \mathrm{mg} / \mathrm{kg})$. We followed-up on significant effects $(P<0.05)$ using the Fisher PLSD post hoc test.

Electrophysiology. The $n$ in the analysis was the total number of neurons and the dependent measure was firing rate of putative dopamine neurons in midbrain. We analyzed the data using the between-subject factor of Virus Condition (KORD, hM4Di) and the within-subject factor of SalB Concentration $(0,100 \mathrm{nM})$.

Fos-IR, KORD-IR, and Fos+KORD Double-IR. We analyzed the data with factorial ANOVA using the betweensubject factors of SalB dose $(0,15 \mathrm{mg} / \mathrm{kg})$ and context (Homecage, Novel).

\section{RESULTS}

Exp. 1: Effect of SalB on Locomotor Activity in Rats Expressing KORD or hM4Di in Midbrain

SalB dose-dependently decreased both spontaneous (saline injections) and cocaine-induced locomotor activity in KORD-expressing rats (Figure 1a). The analysis showed significant main effects of SalB Dose $\left(\mathrm{F}_{(1,7)}=36.9, P<0.01\right)$ and Cocaine Dose $\left(\mathrm{F}_{(1,7)}=5.8, P<0.05\right)$, and an approaching significant interaction between the two factors $\left(\mathrm{F}_{(1,7)}=4.2\right.$, $P=0.08)$. The approaching significant interaction effect is due to the somewhat stronger inhibition of locomotor activity in the cocaine $v s$ the saline condition. In contrast, SalB $(30 \mathrm{mg} / \mathrm{kg}$ ) had no effect on spontaneous or cocaineinduced locomotor activity in hM4Di-expressing rats (Figure 1a). The analysis showed a significant main effect for Cocaine Dose $\left(F_{(1,10)}=32.6, P<0.01\right)$ but not SalB Dose $(P>0.1)$ or an interaction between these two factors $(P>0.1)$. CNO $(3 \mathrm{mg} / \mathrm{kg})$ decreased both spontaneous (saline injections) and cocaine-induced locomotor activity in hM4Di-expressing rats (Table 1). The analysis showed significant main effects of CNO Dose $\left(\mathrm{F}_{(1,7)}=13.0, P<0.01\right)$ and Cocaine Dose $\left(F_{(1,7)}=48.8, P<0.01\right)$, and a significant interaction between CNO Dose and Cocaine Dose $\left(\mathrm{F}_{(1,7)}=10.9, P<0.05\right)$. The significant interaction is due to the stronger inhibition of locomotor activity in the cocaine $v s$ the saline condition.
Table I Effect of CNO on Spontaneous and Cocaine-Induced Locomotor Activity in Rats Expressing AAV-hM4Di in the Midbrain

\begin{tabular}{llc}
\hline & Saline & Cocaine (10 mg/kg) \\
\hline Vehicle & $28 \pm 0.3$ & $158 \pm 26$ \\
CNO $(3 \mathrm{mg} / \mathrm{kg})$ & $17 \pm 0.3^{*}$ & $64 \pm 15^{*}$ \\
\hline
\end{tabular}

Data are mean \pm SEM of meters per $30 \mathrm{~min}(n=9)$. * Different from the CNO condition, $P<0.05$.

\section{Exp. 2: Effect of SalB on Firing Rate of Putative Dopamine Neurons in Midbrain}

SalB completely inhibited cell firing in KORD-expressing but not in hM4Di-expressing putative dopamine neurons (identified by mCitrine fluorescence) (Figures $1 \mathrm{~b}-\mathrm{d}$ ). The statistical analysis, which included 5 KORD expressing neurons and $10 \mathrm{hM} 4 \mathrm{Di}$ expressing neurons, showed a significant interaction between Virus Condition and SalB Concentration $\left(\mathrm{F}_{(1,13)}=14.8, P<0.01\right)$. Additionally, SalB completely inhibited (100\%) cell firing of three KORDinfected neurons in which we did not assess the vehicle condition. Finally, in rats expressing hM4Di in midbrain, bath application of $\mathrm{CNO}(10 \mu \mathrm{M}, n=2,6$ neurons) decreased baseline firing rate by $97.8 \%$.

The in vitro data suggest that KORD activation can suppress neural activity for approximately $15 \mathrm{~min}$ after SalB application (Figure 1c). Additionally, our informal observations of the rats at the end of the locomotor tests were that their locomotor behavior was still suppressed at the end of the session. This suggests that the action of SalB in vivo lasts for at least $30 \mathrm{~min}$.

\section{Exp. 3: Effect of SalB on Novel Context-Induced Fos Expression in Ventral Subiculum Neurons that Project to Nucleus Accumbens Shell}

We observed robust expression of KORD (KORD-IR) in ventral subiculum in rats injected with AAV-Cre into nucleus accumbens shell and AAV-DIO-KORD into ventral subiculum (Figure 2b). This observation demonstrates that the AAV-Cre virus infected ventral subiculum terminals in nucleus accumbens shell, which was then transported back to the cell bodies in ventral subiculum. The statistical analysis showed no differences in KORD-IR between the four groups $(P>0.05)$ (Figure 2c).

SalB injections decreased neuronal activity (as assessed by Fos-IR) in ventral subiculum (Figure 2d). More importantly, these injections also decreased neuronal activity in neurons projecting to nucleus accumbens shell (as assessed by double-labeling of Fos-IR with KORD-IR) (Figure 2e). The analysis of Fos-IR showed a significant interaction between SalB Dose and Context $\left(\mathrm{F}_{(1,19)}=13.4, P<0.01\right)$. The analysis of the double-labeling of Fos-IR with KORD-IR showed main effects for SalB Dose $\left(\mathrm{F}_{(1,19)}=26.6, P<0.01\right)$ and Context $\left(\mathrm{F}_{(1,19)}=7.4, P<0.05\right)$, but no interaction between the two factors $(P>0.1)$ (Figure 2e). The lack of significant interaction illustrates that SalB decreased Fos-IR in KORDIR neurons in both the home-cage and novel context conditions. 


\section{a Retro-DREADD method}

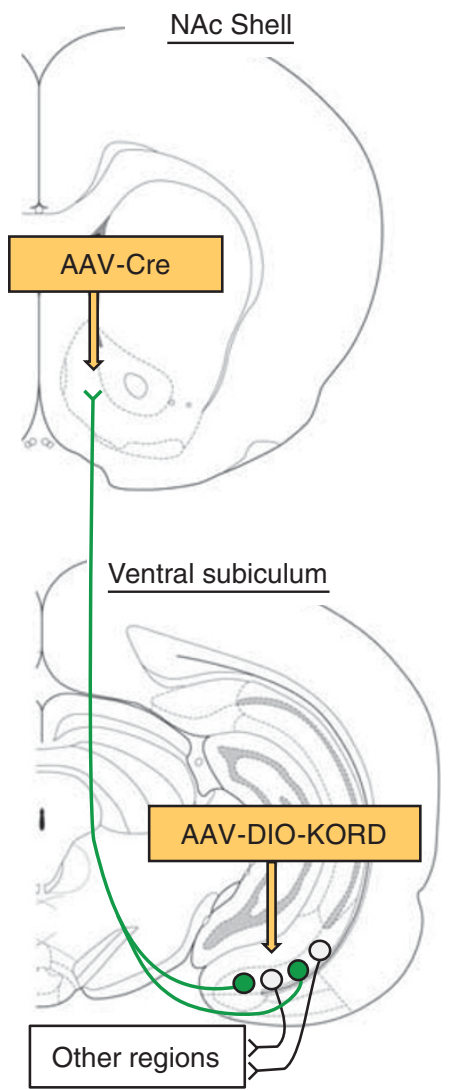

b

Viral expression in accumbens shell and ventral subiculum
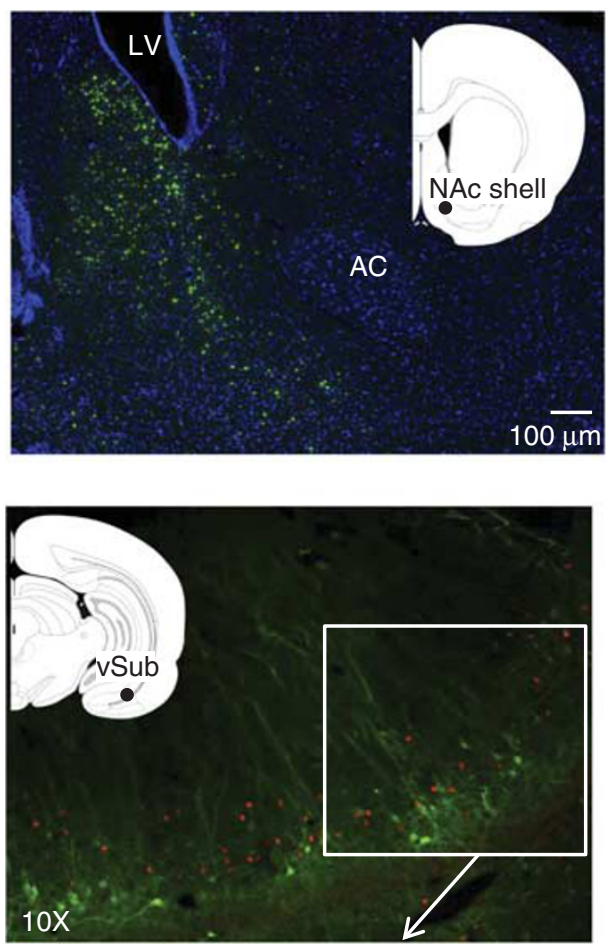

$100 \mu \mathrm{m}$

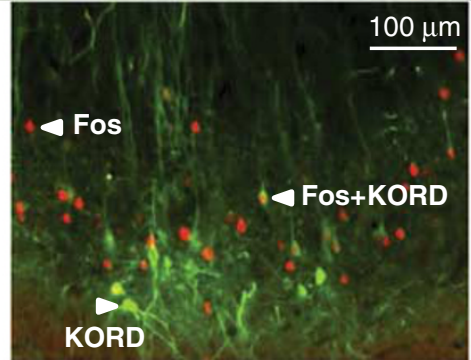

C

Total KORD/mm ${ }^{2}$

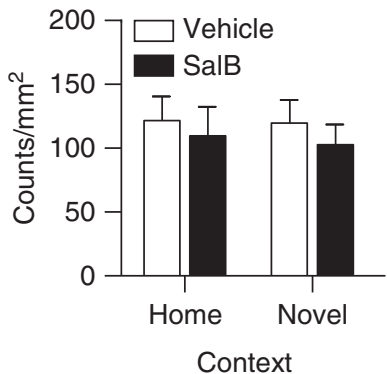

d Total Fos $/ \mathrm{mm}^{2}$

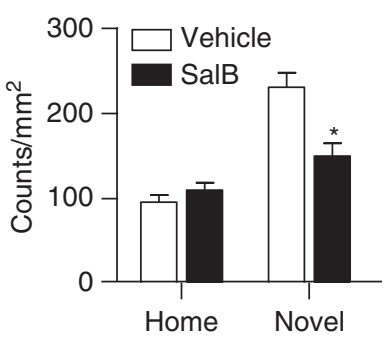

Context
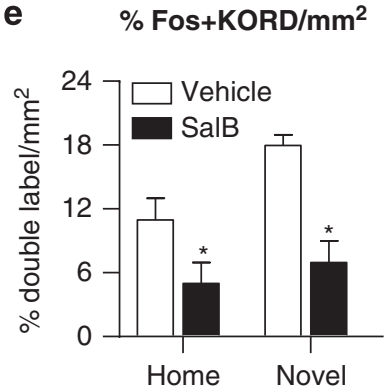

Context

Figure 2 Effect of Salvinorin B on novel context-induced Fos expression in ventral subiculum neurons that project to nucleus accumbens shell. (a) Diagram of retro-DREADD procedure. (b) Representative images of Cre-GFP expression in nucleus accumbens shell and KORD+Fos-IR in ventral subiculum, respectively. AC, anterior commissure; LV, lateral ventricle; Nac, nucleus accumbens; vSub, ventral subiculum. (c) Total KORD/mm²: Number of KORD-IR cells per $\mathrm{mm}^{2}$ in ventral subiculum in rats pretreated with vehicle or SalB $\left(\mathrm{mg} / \mathrm{kg}\right.$ ) and tested in Homecage or a Novel Context. (d) Total Fos $/ \mathrm{mm}^{2}$ : Number of FosIR cells per $\mathrm{mm}^{2}$ in ventral subiculum in rats pretreated with vehicle or SalB $(15 \mathrm{mg} / \mathrm{kg})$ and tested in Homecage or a Novel Context. *Different from vehicle in the Novel Context. (e) \%Fos+KORD/mm²: Percentage of Fos+HA cells per $\mathrm{mm}^{2}$ in ventral subiculum in rats pretreated with vehicle or SalB (I $5 \mathrm{mg} / \mathrm{kg}$ ) and tested in Homecage or a Novel Context. *Different from vehicle in Homecage and Novel Context.

\section{DISCUSSION}

We report three main findings. First, we found that SalB dose-dependently decreased spontaneous and cocaineinduced locomotor activity in rats with KORD expression in midbrain. The effect of SalB on locomotion was selective to KORD-expressing rats as there was no effect of SalB on locomotor activity in rats expressing the Gi/o-DREADD hM4Di in midbrain. Second, we found that SalB completely inhibited cell firing in KORD, but not in hM4Di, expressing neurons. Third, using a 'retro-DREADD' approach (Boender et al, 2014), we showed that SalB decreased novel contextinduced Fos expression in ventral subiculum neurons projecting to nucleus accumbens shell. This observation demonstrates that KORD expression can be restricted to neurons defined by the projection target, and that these neurons can be inhibited in vivo with systemic injections of SalB. This demonstration illustrates the feasibility of using this retro-DREADD approach to study the role of neural circuits in complicated behavioral studies in rats.

Our finding that SalB decreases cocaine-induced locomotor activity in rats expressing KORD in midbrain is consistent with early findings showing that inhibition of the nigrostriatal dopaminergic system decreases psychostimulant-induced motor behaviors (Fibiger et al, 1973; Creese and Iversen, 1975). Our results demonstrate that KORD activation is sufficient to overcome the significant level of behavioral activation induced by cocaine, which 
illustrates the potent effect of KORD activation has to inhibit neuronal activity.

We also showed, using electrophysiology and immunohistochemistry methods, that SalB action on KORD inhibits ex vivo spontaneous action potentials and Fos expression. Additionally, we showed that SalB does not interact with the muscarinic-based DREADD hM4Di in vivo, which is consistent with prior in vitro studies in mice (Vardy et al, 2015). Thus, in the same rat KORD can be used in combination with the muscarinic-based DREADDs (either hM4Di or hM3Dq) to either selectively inhibit two different cell types or neuronal projections (combination of KORD and hM4Di), or to selectively inhibit or excite two different cell types or neuronal projections (combination of KORD and hM3Dq). KORD and hM3Dq can also potentially be used together to inhibit and excite the same cell type or neuronal projection in the same rat, as was demonstrated in mice (Vardy et al, 2015).

Our data provide evidence for restricted expression of Cre-dependent KORD in projection neurons using a retrograde AAV encoding Cre. There is evidence in the literature that certain AAV serotypes have properties that facilitate retrograde transport (Aschauer et al, 2013; Rothermel et al, 2013). The efficiency of retrograde transport is an important consideration in 'Retro-DREADD' studies. Aschauer et al (2013) provided evidence for complete overlap between neurons that were retrograde labeled with AAV and traditional retrograde tracers (cholera toxin $\mathrm{b}$ subunit: $\mathrm{CTb}$ ); however, the total number of AAV-labeled neurons was lower than CTb labeled. Interestingly, we found that the total number of KORD expressing neurons in ventral subiculum was similar to the total number of retrograde labeled neurons identified in ventral subiculum after injections of traditional retrograde tracers, such as CTb or Fluoro-Gold, in nucleus accumbens shell (Marchant, Bossert, unpublished data).

We also provided functional evidence of projection-specific inhibition of neurons using KORD. We found that SalB decreased novel context-induced Fos in ventral subiculum neurons that project to nucleus accumbens shell in rats injected with AAV-Cre into nucleus accumbens shell and AAV-DIOKORD into ventral subiculum. The ventral subiculum sends a strong glutamatergic projection to nucleus accumbens shell (Groenewegen et al, 1987) and hippocampal and tyrosine hydroxylase-labeled terminals in the accumbens converge on the same postsynaptic dendrites (Sesack and Pickel, 1990). We chose to study this particular pathway in the current study because we have shown that both nucleus accumbens shell and ventral subiculum are independently important for contextinduced reinstatement of heroin seeking in our relapse studies (Bossert et al, 2007; Bossert and Stern, 2014).

The current finding suggests that the retro-DREADD dual-virus approach described in our study is suitable for selective projection silencing in studies on circuits controlling appetitive and aversive motivated behaviors in rats. These are studies that cannot be performed or are very difficult to perform in mice. For example, our retroDREADD method is ideally suitable for circuitry-related studies using long-term intravenous drug self-administration procedures (Schuster and Thompson, 1969) and animal models of drug relapse and craving (Shaham et al, 2003; Pickens et al, 2011) that are technically challenging in mice.
The new retro-DREADD method can also be used by investigators who study brain circuits of learning and memory, as assessed in complex learning tasks in rats (Holland and Bouton, 1999; Pearce and Bouton, 2001; Schoenbaum et al, 2009). Additionally, KORDs can be used in combination with the original muscarinic-based DREADDs to permit bi-directional control over either the same or different neural projections as described in Vardy et al (2015). Finally, the dual-virus approach is versatile; one could replace the DIO-KORD construct with other Credependent viruses (eg, Tian et al, 2012; Yang et al, 2013) to monitor and manipulate neural projections during complex behaviors in rats.

\section{FUNDING AND DISCLOSURE}

The authors declare no conflict of interest.

\section{ACKNOWLEDGMENTS}

CNO was obtained from the NIH as part of the Rapid Access to Investigative Drug Program funded by the NINDS. NJM, LRW, JMB, BKH, BTH, KK, SW, and YS were supported by NIDA-IRP funds to the laboratories of Yavin Shaham and Bruce Hope. NJM received support from Early Career Fellowship 1053308 by the National Health and Medical Research Council. TEP was supported by NIH grant DA018151. EV is currently employed at Merck. BLR has consulted in the past 18 months with Pfizer, Novartis, Merck and RuiYi Pharmaceuticals. BLR has received an unrestricted grant from Asubio Pharmaceuticals and receives compensation as Deputy Editor of the Journal of Clinical Investigation. BLR has received grant support for DREADD technology unrelated to this manuscript from Merck Pharmacology. BLR was supported by an NIMH BRAIN Initiative Grant.

\section{REFERENCES}

Alexander GM, Rogan SC, Abbas AI, Armbruster BN, Pei Y, Allen JA et al (2009). Remote control of neuronal activity in transgenic mice expressing evolved $G$ protein-coupled receptors. Neuron 63: 27-39.

Ansonoff MA, Zhang J, Czyzyk T, Rothman RB, Stewart J, Xu H et al (2006). Antinociceptive and hypothermic effects of Salvinorin A are abolished in a novel strain of kappa-opioid receptor-1 knockout mice. J Pharmacol Exp Ther 318: 641-648.

Armbruster BN, Li X, Pausch MH, Herlitze S, Roth BL (2007). Evolving the lock to fit the key to create a family of G proteincoupled receptors potently activated by an inert ligand. Proc Natl Acad Sci USA 104: 5163-5168.

Aschauer DF, Kreuz S, Rumpel S (2013). Analysis of transduction efficiency, tropism and axonal transport of AAV serotypes 1, 2, 5, 6, 8 and 9 in the mouse brain. PLoS One 8: e76310.

Boender AJ, de Jong JW, Boekhoudt L, Luijendijk MC, van der Plasse G, Adan RA (2014). Combined use of the canine adenovirus-2 and DREADD-technology to activate specific neural pathways in vivo. PLoS One 9: e95392.

Bossert JM, Liu SY, Lu L, Shaham Y (2004). A role of ventral tegmental area glutamate in contextual cue-induced relapse to heroin seeking. J Neurosci 24: 10726-10730.

Bossert JM, Poles GC, Wihbey KA, Koya E, Shaham Y (2007). Differential effects of blockade of dopamine D1-family receptors in nucleus accumbens core or shell on reinstatement of heroin 
seeking induced by contextual and discrete cues. J Neurosci 27: 12655-12663.

Bossert JM, Stern AL (2014). Role of ventral subiculum in contextinduced reinstatement of heroin seeking in rats. Addict Biol 19: 338-342.

Bossert JM, Stern AL, Theberge FRM, Marchant NJ, Wang HL, Morales $\mathrm{M}$ et al (2012). Role of projections from ventral medial prefrontal cortex to nucleus accumbens shell in context-induced reinstatement of heroin seeking. J Neurosci 32: 4982-4991.

Boyden ES, Zhang F, Bamberg E, Nagel G, Deisseroth K (2005). Millisecond-timescale, genetically targeted optical control of neural activity. Nat Neurosci 8: 1263-1268.

Creese I, Iversen SD (1975). The pharmacological and anatomical substrates of the amphetamine response in the rat. Brain Res 83: 419-436.

Ferguson SM, Eskenazi D, Ishikawa M, Wanat MJ, Phillips PE, Dong Y et al (2011). Transient neuronal inhibition reveals opposing roles of indirect and direct pathways in sensitization. Nat Neurosci 14: 22-24.

Ferguson SM, Phillips PE, Roth BL, Wess J, Neumaier JF (2013). Direct-pathway striatal neurons regulate the retention of decision-making strategies. J Neurosci 33: 11668-11676.

Fibiger HC, Fibiger HP, Zis AP (1973). Attenuation of amphetamine-induced motor stimulation and stereotypy by 6-hydroxydopamine in the rat. Br J Pharmacol 47: 683-692.

Friedman AK, Walsh JJ, Juarez B, Ku SM, Chaudhury D, Wang J et al (2014). Enhancing depression mechanisms in midbrain dopamine neurons achieves homeostatic resilience. Science 344: 313-319.

Grace AA (1988). In vivo and in vitro intracellular recordings from rat midbrain dopamine neurons. Ann NY Acad Sci 537: 51-76.

Groenewegen HJ, Vermeulen-Van der Zee E, te Kortschot A, Witter MP (1987). Organization of the projections from the subiculum to the ventral striatum in the rat. A study using anterograde transport of Phaseolus vulgaris leucoagglutinin. Neuroscience 23: 103-120.

Holland PC, Bouton ME (1999). Hippocampus and context in classical conditioning. Curr Opin Neurobiol 9: 195-202.

Krashes MJ, Koda S, Ye C, Rogan SC, Adams AC, Cusher DS et al (2011). Rapid, reversible activation of AgRP neurons drives feeding behavior in mice. J Clin Invest 121: 1424-1428.

Morgan JI, Curran T (1991). Stimulus-transcription coupling in the nervous system: involvement of the inducible proto-oncogenes fos and jun. Annu Rev Neurosci 14: 421-451.

Morikawa H, Khodakhah K, Williams JT (2003). Two intracellular pathways mediate metabotropic glutamate receptor-induced Ca2+ mobilization in dopamine neurons. J Neurosci 23: 149-157.

Nair SG, Strand NS, Neumaier JF (2013). DREADDing the lateral habenula: a review of methodological approaches for studying lateral habenula function. Brain Res 1511: 93-101.

Paxinos G, Watson C (2008). The Rat Brain in Stereotaxic Coordinates, 6th edn. Academic Press: San Diego, CA.

Pearce J, Bouton ME (2001). Theories of associative learning in animals. Annu Rev Psych 52: 111-139.
Pennartz CM, Groenewegen HJ, Lopes da Silva FH (1994). The nucleus accumbens as a complex of functionally distinct neuronal ensembles: an integration of behavioural, electrophysiological and anatomical data. Prog Neurobiol 42: 719-761.

Pickens CL, Airavaara M, Theberge FR, Fanous S, Hope B, Shaham Y (2011). Neurobiology of incubation of cocaine craving. Trends Neurosci 34: 411-420.

Rogan SC, Roth BL (2011). Remote control of neuronal signaling. Pharmacol Rev 63: 291-315.

Roth BL, Baner K, Westkaemper R, Siebert D, Rice KC, Steinberg S et al (2002). Salvinorin A: a potent naturally occurring nonnitrogenous kappa opioid selective agonist. Proc Natl Acad Sci USA 99: 11934-11939.

Rothermel M, Brunert D, Zabawa C, Diaz-Quesada M, Wachowiak M (2013). Transgene expression in target-defined neuron populations mediated by retrograde infection with adeno-associated viral vectors. J Neurosci 33: 15195-15206.

Schoenbaum G, Roesch MR, Stalnaker TA, Takahashi YK (2009). A new perspective on the role of the orbitofrontal cortex in adaptive behaviour. Nat Rev Neurosci 10: 885-892.

Schuster CR, Thompson T (1969). Self administration of and behavioral dependence on drugs. Annu Rev Pharmacol 9: 483-502.

Sesack SR, Pickel VM (1990). In the rat medial nucleus accumbens, hippocampal and catecholaminergic terminals converge on spiny neurons and are in apposition to each other. Brain Res 527: 266-279.

Shaham Y, Shalev U, Lu L, De Wit H, Stewart J (2003). The reinstatement model of drug relapse: history, methodology and major findings. Psychopharmacology 168: 3-20.

Tian L, Hires SA, Looger LL (2012). Imaging neuronal activity with genetically encoded calcium indicators. Cold Spring Harb Protoc 2012: 647-656.

Tidgewell K, Harding WW, Schmidt M, Holden KG, Murry DJE, Prisinzano T (2004). A facile method for the preparation of deuterium labeled salvinorin A: synthesis of [2,2,2-2H3]salvinorin A. Bioorg Med Chem Lett 14: 5099-5102.

Ungless MA, Grace AA (2012). Are you or aren't you? Challenges associated with physiologically identifying dopamine neurons. Trends Neurosci 35: 422-430.

Urban DJ, Roth BL (2015). DREADDs (designer receptors exclusively activated by designer drugs): chemogenetic tools with therapeutic utility. Annu Rev Pharmacol Toxicol 55: 399-417.

Vardy E, Robinson JE, Li C, Olsen RHJ, DiBerto JF, Sassano FM et al (2015). A new DREADD facilitates the multiplexed chemogenetic interrogation of behavior. Neuron 86: 936-946.

Whitaker LR, Degoulet M, Morikawa H (2013). Social deprivation enhances VTA synaptic plasticity and drug-induced contextual learning. Neuron 77: 335-345.

Yang CF, Chiang MC, Gray DC, Prabhakaran M, Alvarado M, Juntti SA et al (2013). Sexually dimorphic neurons in the ventromedial hypothalamus govern mating in both sexes and aggression in males. Cell 153: 896-909. 